\title{
A special biotech speculation
}

\author{
Tom Jacobs
}

The classic biotech speculation is a small, development-stage company that possesses breakthrough biotech applied to a drug candidate aimed at a large market. With such a company, the bet is typically all or nothing. Yet what I offer you this month is a company that has, as every parent whose child wants to be a movie star/concert pianist/poet advises, something to fall back on.

Oncology company YM BioSciences (YM; 을 Mississauga, ON, Canada; AMEX:YMI) is not a potential one-hit wonder, but rather has several more tricks up its sleeve. It has not one breakthrough biotech product, but three: tesmilifene, which is being tested in breast cancer and prostate cancer; TheraCIM h-R3 (nimotuzumab), which is in trials for head and neck cancer and for glioma; and AeroLEF (aerosolized liposome-encapsulated fentanyl), which is being tested as a treatment for chronic pain, most commonly from cancer. In short, YM has one drug that clearly addresses large (2) potential markets, and two others that are no slackers.

\section{The trailblazer}

Tesmilifene is a novel and controversial smallmolecule chemopotentiator, a treatment to improve the effectiveness of chemotherapy in cancer patients. In its first phase 3 trial for aggressive breast cancer, tesmilifene did not shrink tumors yet resulted in signs of increased overall survival-a 50\% increase compared with a 25\% for Genentech's (S. San Francisco, CA, USA; NYSE:DNA) Herceptin (trastuzumab). This quandary led to the discovery that tesmilifene sensitizes multidrug resistant cells to chemotherapy, by inhibiting, rather than accelerating, the P-glycoprotein pump. ATP is consumed and apoptosis follows.

Tom Jacobs is cofounder of Complete Growth Investor and can be contacted via http://www. completegrowth.com, a stock service for individual investors. YM Biosciences is a Complete Growth Investor selection and Tom owned shares at time of writing
Decades of research has emphasized tumor shrinkage as a primary endpoint in cancer therapy. Tesmilifene potentially overturns that paradigm, which is why it is a tad controversial. Nonetheless, results have been sufficiently impressive to snag big pharma interest; YM is currently partnering with Sanofi-Aventis (Paris; NYSE:SNY) in pivotal phase 3 testing of tesmilifene against aggressive breast cancer. By the end of the year, it hopes to have enough data to file a New Drug Application with the US Food \& Drug Administration (FDA). A phase 2 trial for prostate cancer is two-thirds enrolled and another is approved, but not yet initiated, for gastric cancer in collaboration with Seoul, Korea-based Shin Poong Pharmaceuticals.

\section{The follow-up}

Next in the pipeline is TheraCIM, a humanized monoclonal antibody antagonist of epidermal growth factor receptor (EGFR), which originated from the University of Havana, Cuba. Granted orphan drug status by the FDA and European Medicines Agency, TheraCIM is in testing to treat pediatric brain cancer.

Like tesmilifene, TheraCIM is somewhat controversial. Many cancer patients receiving other antibody antagonists of EGFR have reported a painful rash. According to YM's CEO David Allen, some patients would rather endure the condition itself than take anti-EGFR drugs and have the rash. OSI Pharmaceuticals (Melville, NY, USA: Nasdaq:OSIP) and ImClone Systems (New York: Nasdaq:IMCL) claim that the rash indicates benefit. But to date, TheraCIM, which also targets EGFR, has shown efficacy without evidence of eliciting rash in patients in clinical trials, nor any other side effects, such as brittle hair, conjunctivitis and toenail inflammation. The drug is in numerous phase 2 and trials.

One potential obstacle is the drug's origin at the University of Havana and the United States' trade embargo with Cuba. However, several drugs have been quietly granted US waivers from trade restrictions and YM expects the same for TheraCIM should it get to that stage.

\section{Third string to the bow}

The other lead drug in YM's quiver is AeroLEF, an aerosolizing delivery system for the opioid painkiller fentanyl; it too presents a potential breakthrough. Currently, the top methods of delivering fentanyl, such as Cephalon's (Frazier, PA, USA; Nasdaq:CEPH) Actiq berry-flavored 'lollipop' provide a fixed dose of the opioid to the buccal mucosa. The problem is that the variability in response to an opioid dose is huge, the consequences of excessive opioid dosing are severe, and underdosing means more pain. AeroLEF addresses these issues in two special ways: patient ability to control the dosing, and immediate effect and sustained release.

Yet even if AeroLEF were to be approved, no one should underestimate the difficulty of selling into the chronic pain market. Regulatory concerns are great, particularly given the potential for a treatment to crossover into the general market for addiction, such as OxyContin (oxycodone). Investors in Ligand Pharmaceuticals (San Diego; LGND.PK) know that its product Avinza (morphine sulfate capsules) provides sustained release and less frequent dosing than morphine, but still has failed to provide enough sales to lift Ligand into profitability.

\section{Finances and acquisitions}

YM has plenty of cash and no debt, but will need to raise more to fund its development. Investors should expect more dilution as the company sells additional shares for R\&D and possible acquisitions.

YM's most recent acquisition, privately held Eximias (Berwyn, PA, USA), for \$75 million, gives this Canadian company a base in the world's most lucrative oncology market.

With numerous trial and regulatory milestones in the coming year, success in one or more would make YM, with its \$180-million enterprise value, a very different company. YM indeed is a special biotech speculation for those wishing to make one. 